\title{
Homeostatic brain network mechanisms facilitate perceptual binding of audio-visual speech
}

\author{
Abhishek Mukherjee ${ }^{\dagger}$, Soibam Shyamchand Singh ${ }^{\dagger}$, Dipanjan Ray, \\ Partha Raghunathan, and Arpan Banerjee* \\ National Brain Research Centre, NH8, Manesar, Gurgaon 122052, India. \\ ${ }^{\dagger}$ These authors contributed equally to this work (co-first authors). \\ *To whom correspondence should be addressed. E-mail: arpan@nbrc.ac.in.
}

In daily lives, speech perception requires binding of spatiotemporally disjoint auditory and visual cues. Understanding the associated brain information processing requires characterization of the two complementary network mechanisms, functional segregation and integration. Here, we demonstrate using fMRI recordings, during perception of congruent and incongruent (McGurk) speech stimuli, that subjective perceptual experience of multisensory speech stimuli is dependent on a homeostatic balance of segregation and integration mechanisms. We were able to parametrically control the inter-subject variability of illusory perception by introducing temporal lags in the incongruent auditory-visual articulations of speech sounds. Enhancement of activity as a function of propensity to perceive illusion is observed in distributed brain regions, defined as the perceptual binding network whose anatomical locations range from sensory to associative cortices. Reduction in global integration can be inferred from the anti-correlation observed between the seed-based whole-brain functional connectivity computed at each node of the perceptual binding network and the propensity for illusory perception. That enhanced segregation and reduced integration facilitates cross-modal binding was further validated by the increase in local clustering and modularity, concurrently with lowering in participation coefficient. Further, the perceptual binding network was anticorrelated with other intrinsic functional brain networks, such as dorsal attention and default mode networks during cross-modal perception. The degree of activation and anti-correlation decreased for people who reported lower illusory perception, further strengthened the hypothesis of homeostatic balance.

\section{Keywords}

multisensory, whole-brain functional connectivity, pSTS, fMRI, segregation, integration, homeostasis, perception, predictive coding, Bayesian 


\section{Introduction}

The illusion of a puppet vocalizing human speech that in reality comes from the sounds of a ventriloquist has intrigued mankind for ages (Alais and Burr, 2004). While spatial incongruency is a well-studied aspect of ventriloquism, perceptual binding of temporally dissonant stimuli is subtle (Bertelson and Aschersleben, 2003), such as when viewing a poorly dubbed video our perceptual system immediately perceives the asynchrony in sound and lip movements even when we do not know the language. Some previous studies have also reported that temporal dissonance within a certain window between multiple sensory components can be integrated to facilitate illusory perceptual experience (Bertelson and Aschersleben, 2003; Munhall et al., 1996; Shams et al., 2002; Stevenson et al., 2012), but do collective behavior of brain networks exhibit patterns through which integrative mechanisms of brain information processing underlying perceptual binding can be understood is an unanswered question.

In this article, we hypothesized that cooperation among relevant brain areas via functional integration mechanisms (Friston et al., 1993) and their individual role in information processing need to be at a critically balanced state to facilitate cross-modal perceptual binding, and this relationship may be parametrically controlled by behavioral contexts. Previous studies have used illusory perceptual experience from the paradigms of sound-flash illusion (Watkins et al., 2007) and McGurk illusion (McGurk and Macdonald, 1976) to suggest posterior superior temporal sulcus (pSTS) as the key hub for cross-modal percept binding (Beauchamp et al., 2004; Nath and Beauchamp, 2011), whereas other studies have not found pSTS activations to be enhanced specific to temporal synchrony of auditory and visual modalities (Jones and Callan, 2003). From a large-scale network perspective, noisy stimulus in one modality has been shown to increase functional brain connectivity between pSTS and areas processing in the other modality; for example, a blurry video will increase strength of pSTSauditory connectivity (Nath and Beauchamp, 2011). Such shifts in connectivity arising from stimulus associations can be linked to causal inference models of perception (Koerding et al., 2007; Magnotti et al., 2018) and lately biophysically realistic network mechanisms have been proposed (Cuppini et al., 2017; Kumar et al., 2020; Thakur et al., 2016). Audio-visual speech perception also involves active participation of the motor network (Dehaene-Lambertz, Ghislaine and Hertz-Pannier, Lucie and Dubois, Jessica and Mériaux, Sébastien and Roche, Alexis and Sigman, Mariano and Dehaene, Stanislas, 2006; Liberman, Alvin M and Whalen, Doug H, 2000; Morís Fernández, L. and Macaluso, E. and Soto-Faraco, S., 2017; Scott, S. K and McGettigan, C. and Eisner, F., 2009), and there is increased interaction between motor representational area and inferior frontal gyrus during presentation of incongruent (McGurk) speech stimuli, as compared to congruent ones. This motor network was found to contribute toward resolving the audiovisual conflicts, thus avoiding the McGurk perception (Murakami, T. and Abe, M. and Wiratman, W. and Fujiwara, J. and Okamoto, M. and MizuochiEndo, T. and Iwabuchi, T. and Makuuchi, M. and Yamashita, A. and Tiksnadi, A. and others, 2018). The manifestation of audio-visual illusory speech perception can thus be viewed as a critical balance between functional domains promoting the integration (such as the pSTS network) and domains with more abstract functions involved in conflict resolution (such as the motor network). Finally, existence of a homeostatic balance in network mechanisms can explain why perception is relatively stable for a wide range of behavioral contexts (Zopf Jr, G. W, 1963), e.g., perception of illusory sound in the McGurk paradigm for different vocalizers and post-learning (Mallick, D. B. and Magnotti, J. F. and Beauchamp, M. S., 2015), perceivers across cross-cultural boundaries (Magnotti, J. F. and Mallick, D. B. and Feng, G. and Zhou, B. and Zhou, W. and Beauchamp, M. S., 2015), even though inter- 
individual variability exist. An empirical demonstration of how subjective perceptual binding emerges from synergistic functional integration and segregation mechanisms in brain networks (Tomasi et al., 2014) is currently elusive.

To elicit instances of cross-modal perceptual experience, the McGurk incongruent video-auditory /pa/ superimposed on the lip movement of /ka/ from a human vocalizer (McGurk and Macdonald, 1976) — was shown to volunteers. Subsequently, subjective perception was parametrically controlled by using different versions of this video that were constructed with temporal lags introduced between the auditory and visual sensory streams ranging from -300 (A leading V) to $450 \mathrm{~ms}$ (V leading A) in intervals of $150 \mathrm{~ms}$ (Fig. 1A), along with a matched number of congruent /ta/ (auditory /ta/-visual $/ \mathrm{ta} /)$ presented at $0 \mathrm{~ms}$ lag. The choosing of an asymmetrical range of audio-visual (AV) lags ( $-\mathrm{L} 2$, $\ldots, \mathrm{L} 0, \ldots, \mathrm{L} 3$ ) was guided by findings from a previous study (Munhall et al., 1996) reporting about a bias in perceptual experience toward visual-leading scenarios. Volunteers reported whether they heard /ta/, /pa/, or "others" in a forced-choice paradigm. Thus, a behavioral control of subjective illusory /ta/ perception, hallmark of cross-modal perceptual binding, was achieved with AV lags as the control parameter (Fig. $1 B$ and SI Appendix, Figs. S1 and S2). To understand the underlying mechanism, the fMRI data were analyzed through both stimulus-driven as well as perceptual-based approaches (see Materials and Methods for details).

Identifying integration and segregation characteristics (Tomasi et al., 2014; Tononi et al., 1994) of large-scale brain networks is increasingly becoming an important tool to understand information processing mechanisms underlying various cognitive tasks (Cohen and D'Esposito, 2016; Mohr et al., 2016). A more riveting hypothesis is that normative human cognition can be defined as a homeostatic balance between integrative and segregative mechanisms with imbalances leading to neuropsychiatric disorders (Lord et al., 2017). The present study demonstrates how such a balance facilitates perceptual binding of incongruent audio-visual/multisensory stimuli. This is a significant departure from the earlier understanding of multisensory integration from the perspective of inverse effectiveness which has been reinforced by several studies using different neuroimaging modalities (Meredith and Stein, 1983; Molholm et al., 2002; Stevenson and James, 2009).

\section{Results}

Parametric Control of Illusory Perception. More than chance level responses to illusory /ta/ (cross-modal perception) were observed in all participants at multiple AV lags for the McGurk incongruent stimulus, demonstrating that indeed the degree of AV temporal synchrony affects subjective perception (Fig. 1 and SI Appendix, Figs. S1 and S2). In order to study the neural basis of subjective variability, we categorize the participants into two equally sized groups $(N=19)$ while sorting them based on their susceptibility to audio-visual illusion. The susceptibility is measured as the average of two maximal percentage /ta/ responses in any two AV lags. The group reporting higher illusory perception is termed as high perceivers and the other group at the lower end as low perceivers (SI Appendix, Fig. S1 $A$ and $B$ ).

Analysis of variance (ANOVA) performed on high perceivers $(N=19)$ revealed that response percentage of illusory /ta/ was significantly varying at $95 \%$ confidence levels (a confidence interval used in all statistical significance tests in the article) across AV lags $(F(5,106)=12.8564, p<$ 0.0001). High perceivers also showed significant variation across AV lags for unisensory /pa/ responses $(F(5,106)=4.7753, p=0.0005)$ as well as for “other" responses $(F(5,106)=2.7982, p=0.0205)$. On the other hand, low perceivers $(N=19)$ showed significant variation only for cross-modal /ta/ perception $(F(5,107)=5.3264, p=0.0002)$ but not for $/ \mathrm{pa} /(F(5,107)=2.1205, p=0.0684)$ or 


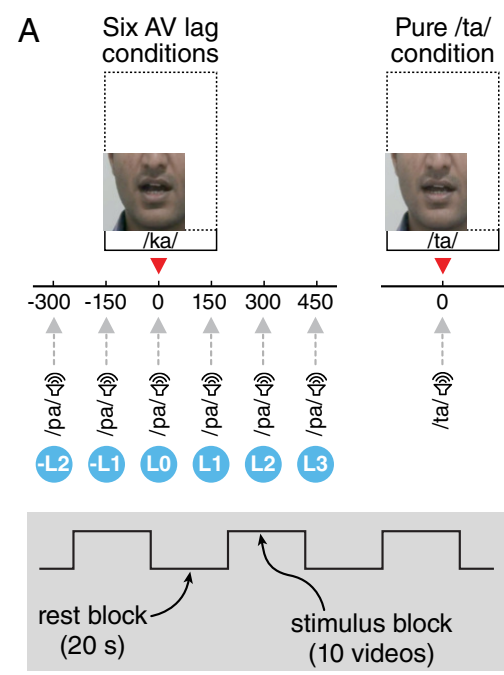

B all 38 selected volunteers

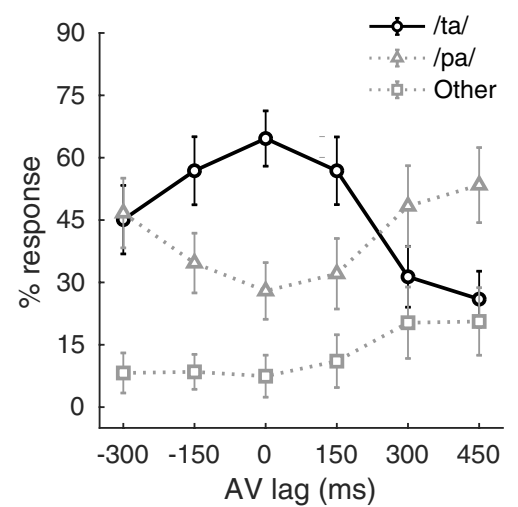

C high perceivers

D low perceivers
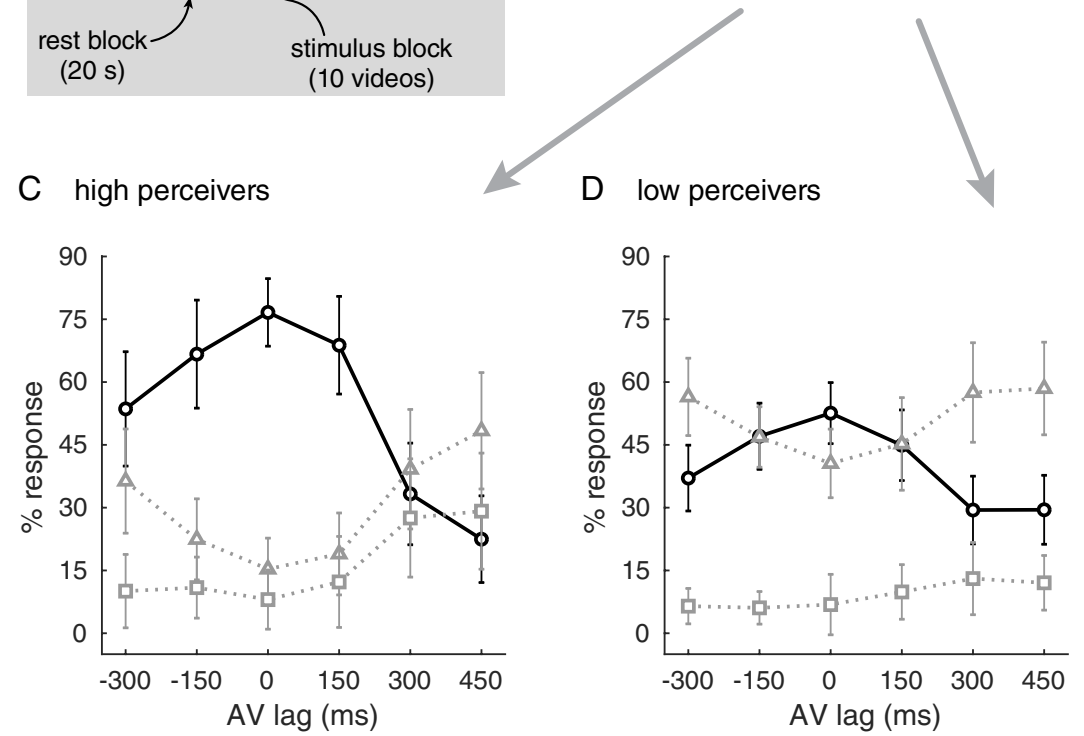

Figure 1. (A) Stimulus design of the study. $(B)$ Behavioral responses averaged across all 38 selected volunteers. Behavioral responses averaged across $(C)$ high perceivers $(N=19)$ and $(D)$ low perceivers $(N=19)$. Error bars represent the standard error of the mean $(95 \% \mathrm{Cl})$ across the respective population.

“other" responses $(F(5,107)=0.8144, p=0.5419)$. Welch's t-test was further performed to investigate the differences in perceptual responses between high and low perceivers for each AV lag condition. Significant differences in illusory /ta/ perception between high and low perceivers occurred at $-\mathrm{L} 1(p=0.0193), \mathrm{L} 0(p=0.0001)$, and L1 $(p=0.0033)$. Unisensory /pa/ perception also showed significant differences at $-\mathrm{L} 2(p=0.0194),-\mathrm{L} 1(p=0.0004), \mathrm{L} 0(p=0.0001)$, and L1 $(p=0.0017)$ (SI Appendix, Fig. S1C). For the "other" response, significant difference was observed only at L3 $(p=0.0429)$.

Brain Mapping of Perceptual Binding. Analysis of fMRI images using statistical parametric mapping (SPM) software (Friston et al., 1995) was performed with the task (AV lag blocks) and baseline (inter-trial rest blocks) conditions. Group-level inference from random effect analysis revealed that maximal brain areas were active during LO and L1 conditions, where propensities to cross-modal illusory perception were also maximally reported (Fig. 2). The extent of task-activated brain areas diminished bilaterally with increasing AV separation (Fig. 2 and SI Appendix, Tables S1 and 
high perceivers

-L2
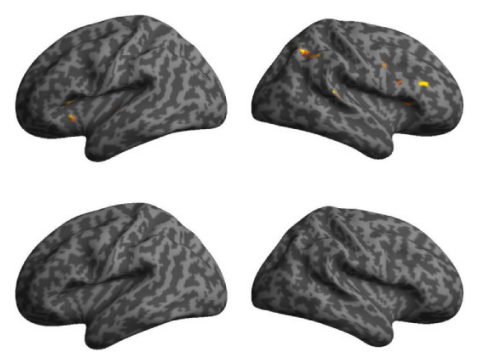

L0
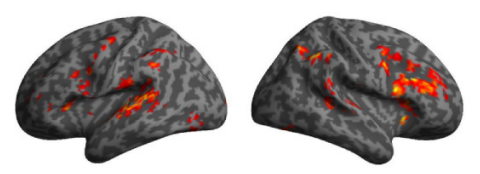

L1
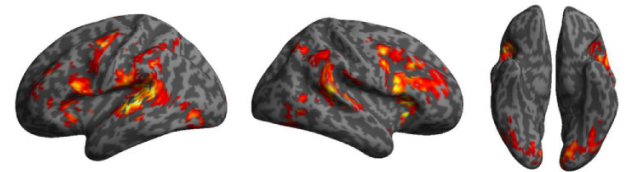

L2
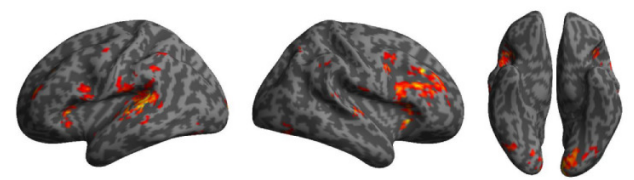

L3
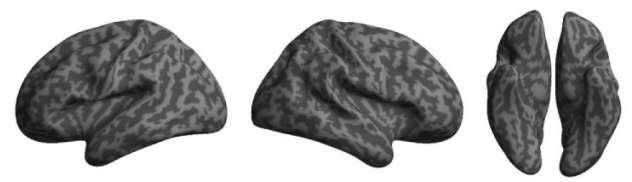

Pure /ta/
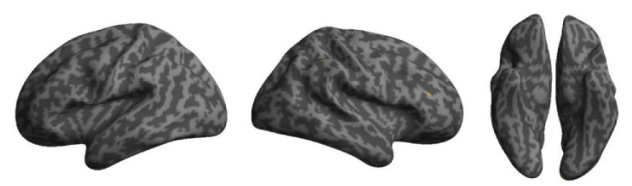

$\operatorname{Min} / \mathrm{ta} /$
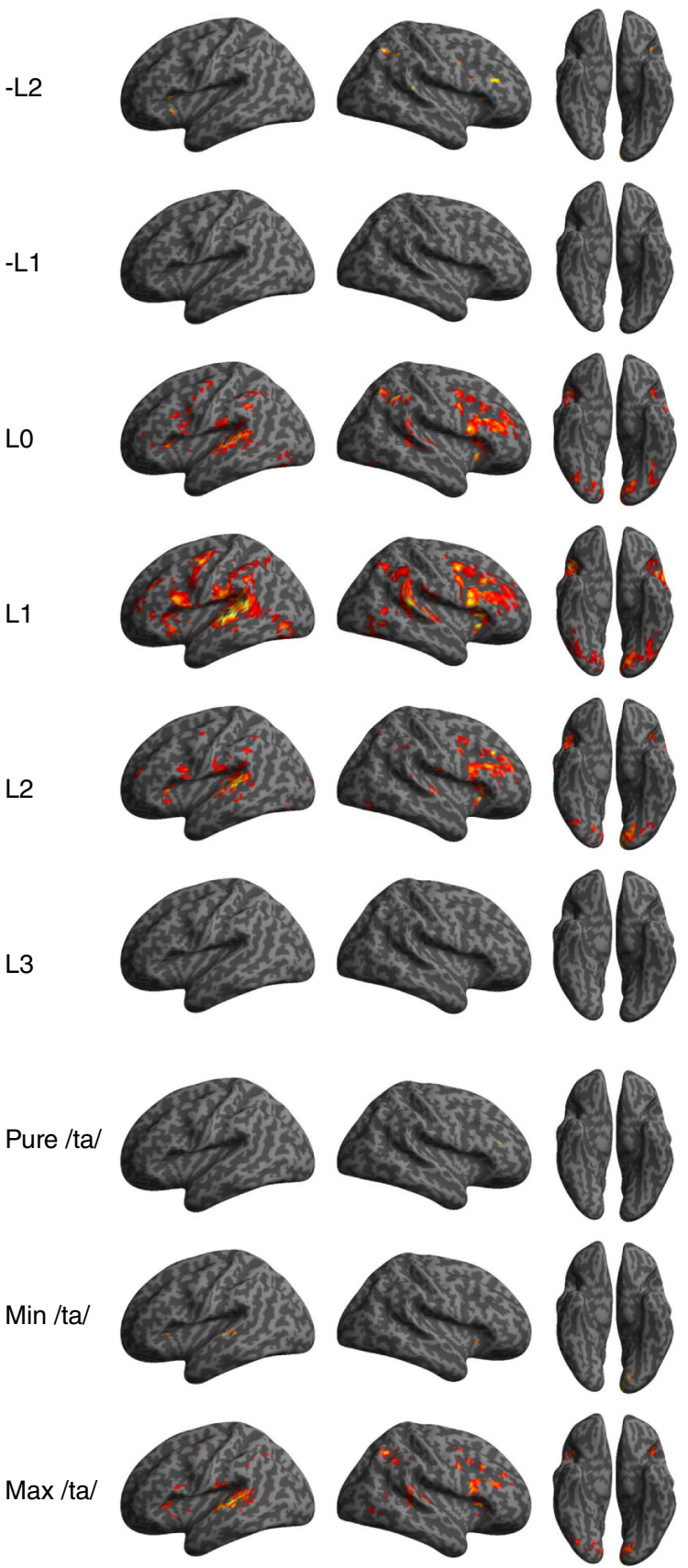

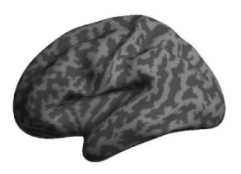

low perceivers
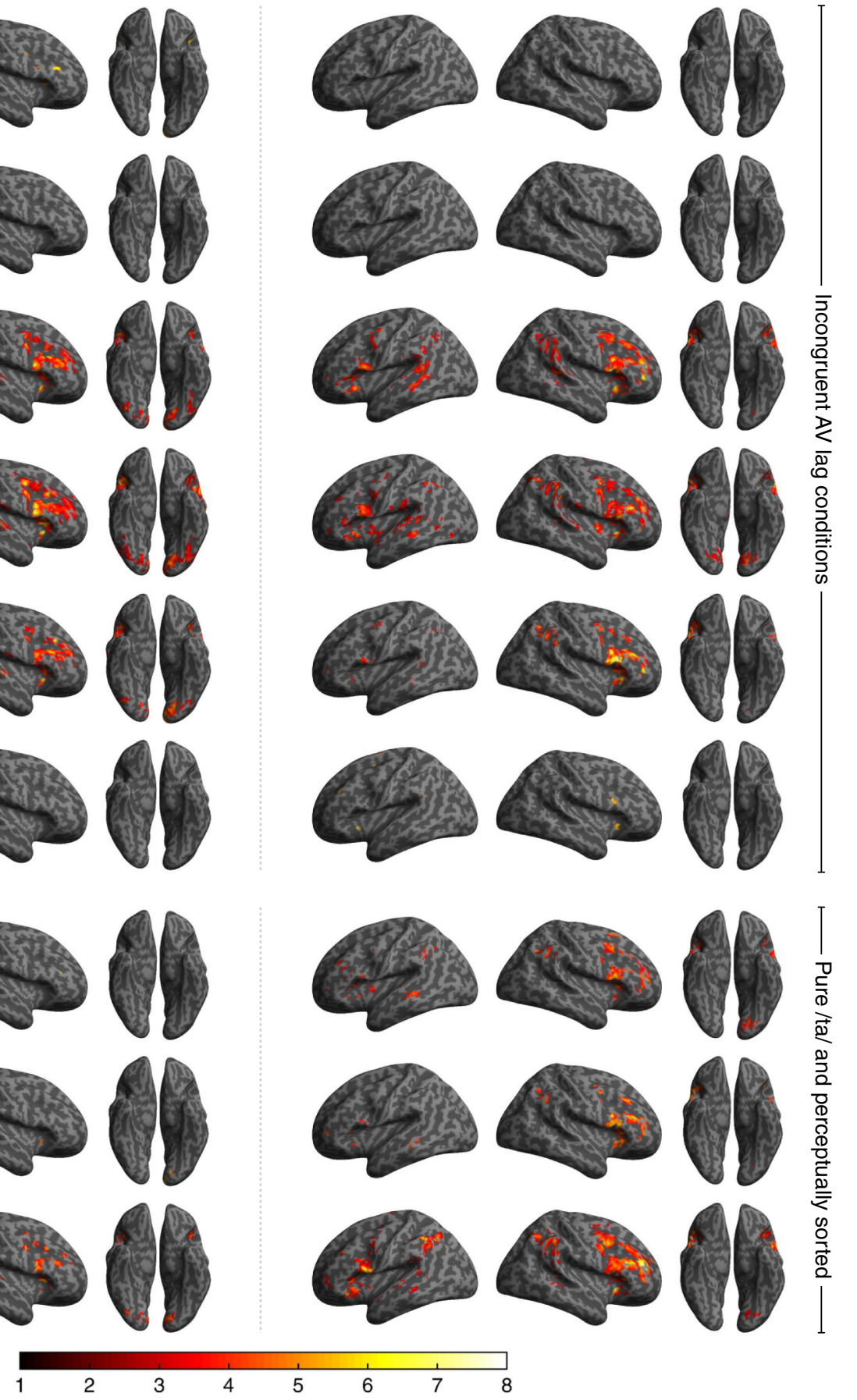

Figure 2. Group-level task-enhanced (task $>$ rest) active brain areas for high and low perceivers (peak $p \leqslant 0.05$, FDR corrected). Color bar represents the T-score.

S2). Among those perceptual-sorted categories, Max /ta/ condition showed maximal brain activation. Furthermore, when compared with high perceivers, the low perceivers showed relatively weaker activation specially in primary motor, right frontal, anterior cingulate cortex, superior temporal region including pSTS, rostral inferior parietal, and occipital areas: on the other hand, orbitofrontal, insula, and thalamic regions showed higher activation (see SI Appendix, Table S2). 
To study the differential functional roles of associated brain regions during audio-visual crossmodal perception, 32 seed regions of interest (ROIs) were defined using another independent subset of 29 participants, each of whom reported $60 \%$ or more maximal illusory /ta/ response in at least one AV lag condition (SI Appendix, Tables S3 and S4; see also Materials and Methods for more details). We will henceforth term these 32 seed ROIs which were together active during the audio-visual illusory (cross-modal) percept formation as the perceptual binding network (PBN). The number of active voxels and percentage signal difference (PSD) were estimated in all ROIs of PBN for both high and low perceivers (SI Appendix, Figs. S3-S6). Peak PSDs for high perceivers were observed at either L0 or L1 condition (except for bilateral MVOcC and thalamus), and at Max /ta/ condition (except for left MFG, bilateral ACC, MVOcC, and thalamus). Moreover, for low perceivers, relatively higher PSDs were observed toward more video-leading stimulus conditions, and peak values were observed at either L1 or L2 (SI Appendix, Fig. S6). When compared with the high perceivers, low perceivers showed overall decrease of PSDs bilaterally in STG and LOcC, and in left MVOcC. Left hemispheric ROIs, such as MFG, IFG, FuG, IPL, and bilateral pSTS also displayed decreased PSD at L0, L1, and L2. Conversely, the seed region in right PrG area showed overall increase in PSD. Interestingly, PSDs for low perceivers were rather invariant across AV lags for the video-leading conditions, and its value during Max /ta/ were also more comparable to those of unisensory Pure /ta/ condition (SI Appendix, Fig. S6).

Synergistic Network Organization. To investigate the functional integration mechanisms in the 32 seed regions of PBN, the seed-based whole-brain functional connectivity (SWFC) was computed from parcellated time series (see Materials and Methods for details). The averaged SWFC decreased maximally at L0 followed by L1 (Fig. $3 A$ and $C$ shows example pSTS seeds). For high perceivers, among perception-sorted categories, Max /ta/ exhibited lowest SWFC followed by Min /ta/ and Pure /ta/ (Fig. 3B). However, for low perceivers, Max /ta/ and unisensory Pure /ta/ displayed comparable SWFC values (Fig. 3D). Additionally, the spatial organization of SWFC with pSTS parcels as seed regions is also shown in SI Appendix, Figs. S7 and S8. The question now is whether this decrease in SWFC can be meaningfully associated with the propensity for illusory perception. To meet this end, SWFC-behavior correlation (SBC; see Materials and Methods) was computed for each PBN seed regions. Interestingly, for high perceivers all the 32 parcels displayed negative SBC, which means the SWFC as a function of AV lags is anti-correlated with the propensity for illusory $/ \mathrm{ta} /$ perception. Seed ROIs in primary motor areas, left inferior frontal region, superior temporal region including pSTS, inferior parietal area, and lateral occipital area displayed the strongest anti-correlation (SI Appendix, Fig. S9 and Table S5). In the case of low perceivers, several seed regions displayed positive SBC values or comparatively weaker negative SBC (SI Appendix, Fig. S9 and Table S5). When the participants are regrouped into three smaller categories (high perceivers, $N=13$; medium perceivers, $N=12$; low perceivers, $N=13$; see also SI Appendix, Fig. S2B), the SBC values also mostly scaled with the propensity for illusory perception in each category (SI Appendix, Fig. S10). This also further emphasized that globally decreased information integration through the PBN seed regions was essential for the illusory audio-visual percept formation.

To further address the main hypothesis that perceptual binding is effectuated by a homeostatic balance of network mechanisms, functional segregation and integration, we focus on the topological features of the whole-brain functional network using metrics from graph theory. 50 binarized wholebrain network subsamples were constructed for each stimulus condition from random permutations of $70 \%$ of the participants in each group of perceivers - high and low. For estimating the network segregation, averaged local clustering coefficient and modularity measures were used; the integration measures include the characteristic path length and averaged node participation coefficient. Each network 


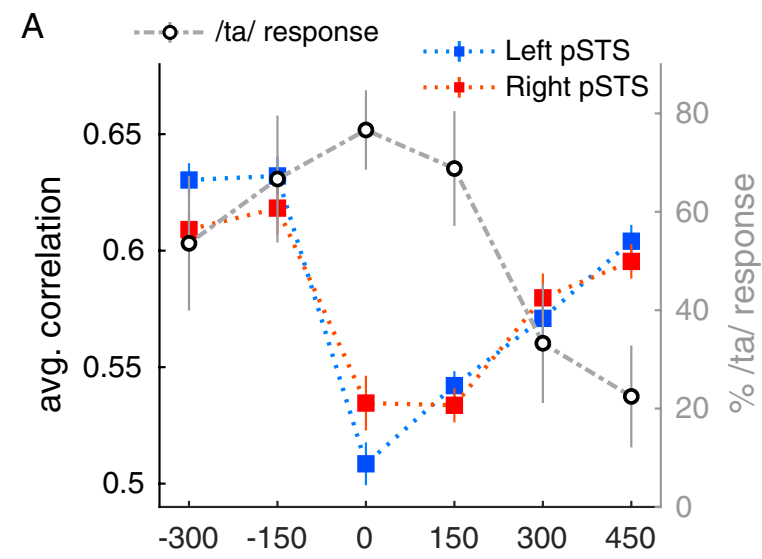

C

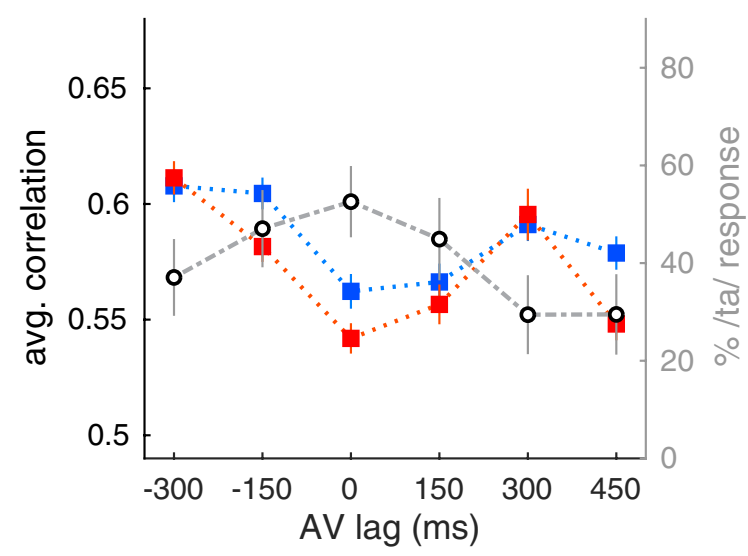

B

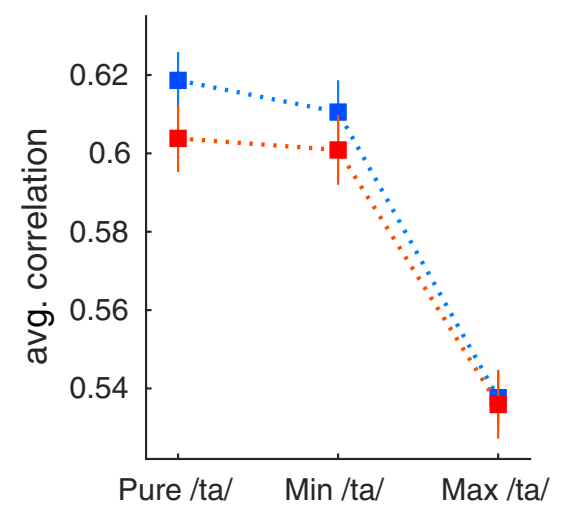

D

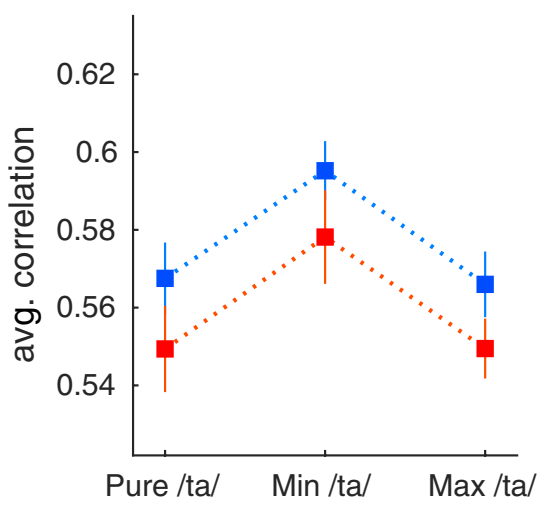

E

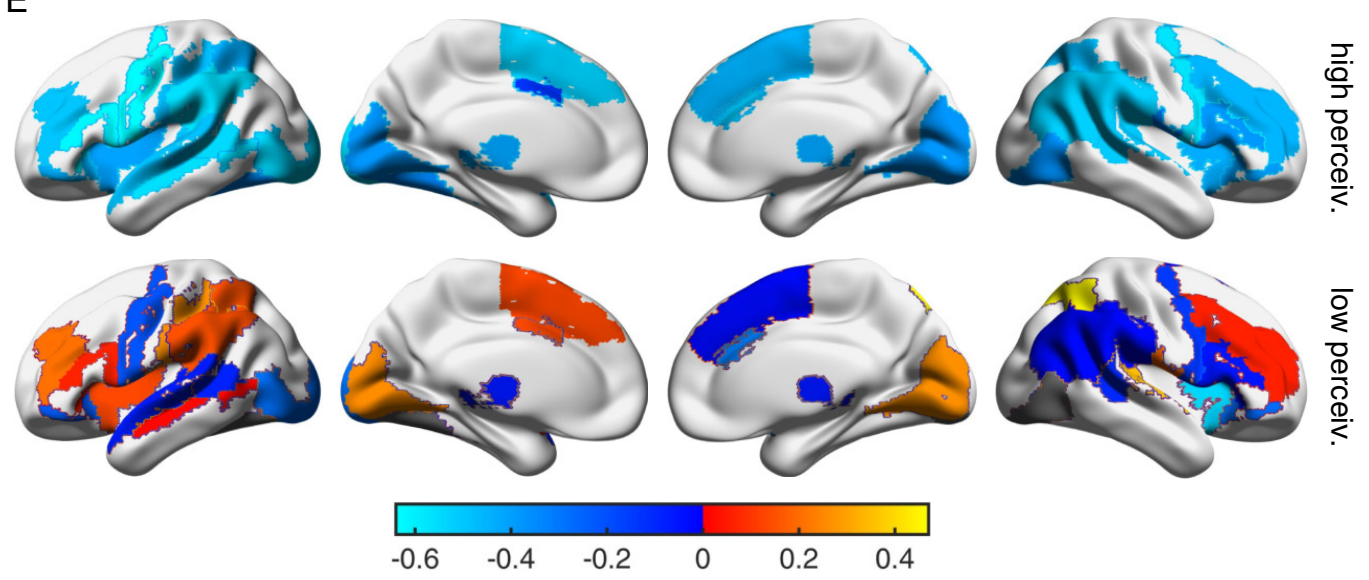

Figure 3. Average seed-based whole-brain functional connectivity (SWFC) of example pSTS seeds for high perceivers: AV lag conditions $(A)$, and Pure /ta/ and perceptually sorted categories $(B)$. Panels $(C)$ and $(D)$ represent the corresponding pSTS seed average SWFC across low perceivers. Error bars represent the standard error of the mean $(95 \% \mathrm{Cl})$. (E) Surface plot showing all 32 seed ROls of the PBN, color mapped according to SWFC-behavior correlation (SBC) values for the high perceivers (upper) and low perceivers (lower) (SI Appendix, Table S5; see also Materials and Methods for details). 
measure was further normalized with estimated measures from randomized networks (see Materials and Methods for more details). In the case of high perceivers, the normalized clustering coefficient $\left(C_{n}\right)$ increased with decreasing $\mathrm{AV}$ lag and peaked at L0 where the reported average propensity to audio-visual illusory perception is the highest (Fig. 4A). This indicates that there is increased local cohesiveness in the network with increasing illusory perception. However, contrary to this effect is the increase in normalized characteristic path length $\left(L_{n}\right)$, reflecting to decreased global cohesiveness. The manifestation of audio-visual illusion might thus require a critical balance between the segregrative (local cohesion) and integrative (global cohesion) information processing. Consequently, higher degree of illusory experience is associated with increasing dissociation among the functional modules, as reflected from the increased normalized modularity score $\left(Q_{n}\right)$. The functional dissociation was also reflected from decrease in average node participation coefficient $(P)$ with increased illusory perception (Fig. 4A). Thus, the balance between functional segregation and integration is manifested at the node-level measure (e.g., local clustering coefficient, participation coefficient), and also at the whole-network-level measure (e.g., normalized modularity score). Low perceivers also displayed a similar trend, however the maximum values of clustering coefficient, characteristic path length, and modularity, and also the minimum value of participation coefficient were observed in L1 condition. The network differences between high and low perceivers is summarized in Fig. $4 B$ which displays only the left hemispheric brain networks at L0 condition: for example, the modules in the frontal and temporal brain regions show increased within-module connectivity both in number and strength (cf. blue arrow), and decrease in-between-module connectivity (cf. magenta and black arrows). However, except for the L0 condition, the increased illusory effect in high perceivers could not be interpreted from the difference in the estimated network measures. One possible explanation for this inconsistency is the difference in the activation patterns between the two groups. More specifically, reduced activation was observed in many sensory as well as associative cortical regions of low perceivers, and this difference in activation pattern might shift the segregation-integration balance accordingly. The graph theoretic measures for high perceivers overall delivered a similar take-home message as observed for SWFC with behavior, that LO condition triggers the peak of alteration in homeostatic balance of network mechanisms realized via the peak of clustering coefficient, characteristic path length, and modularity; and also of minimum participation coefficient. When sorted across perceptual categories Max /ta/ was always significantly different from Pure /ta/ in all the estimated network measures: clustering coefficient $(p<0.0001)$, characteristic path length $(p<0.0001)$, modularity $(p<0.0001)$ and participation coefficient $(p<0.0001)$. In the case of low perceivers, the graph theoretic network metrics were similar between Max /ta/ and Pure /ta/, and no significance differences were obeserved especially for the clustering coefficient $(p=0.2626)$ and modularity $(p=0.0747)$. 

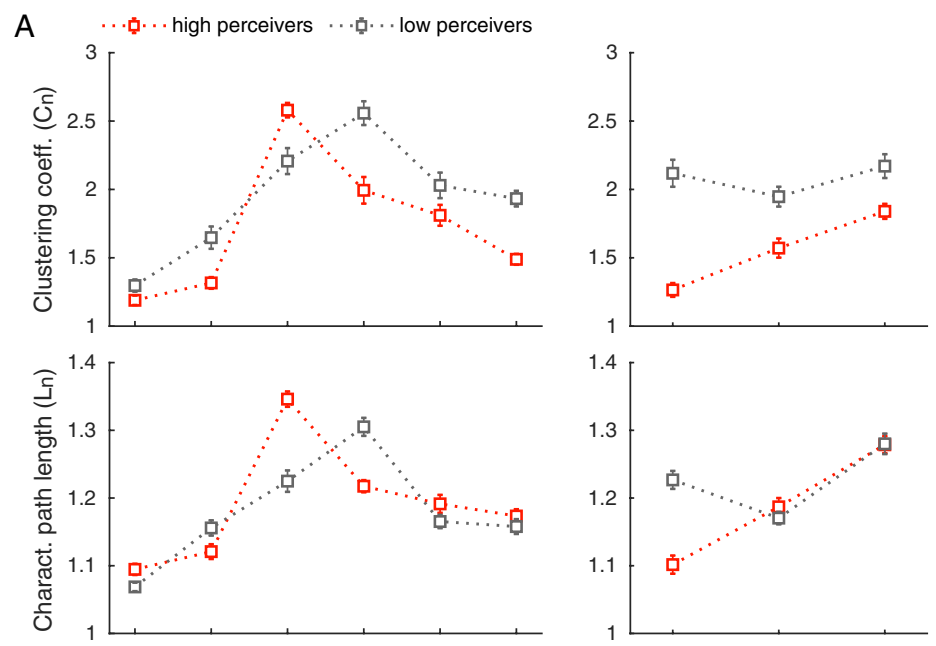

B
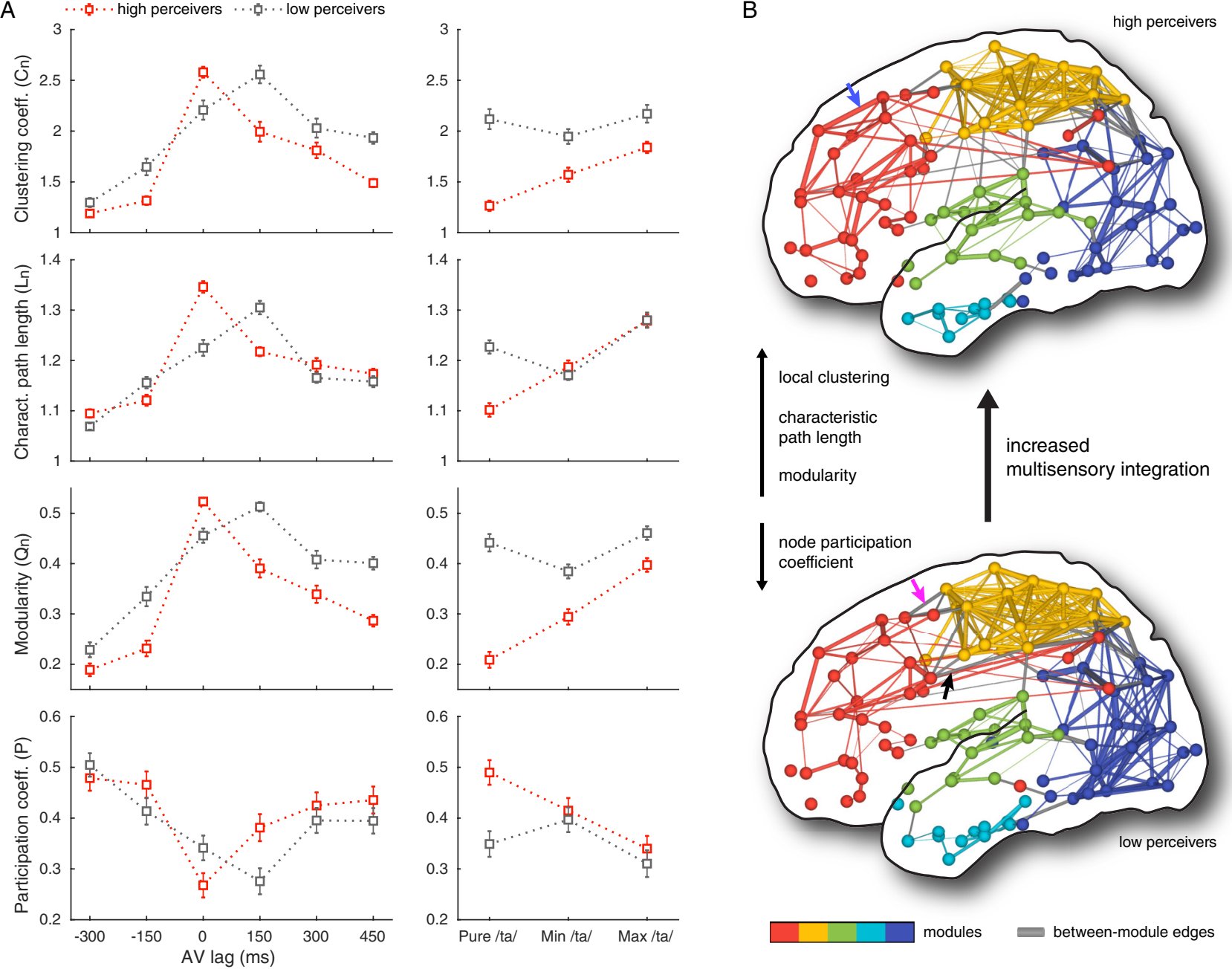

Figure 4. $(A)$ Change in the network topological measures for high and low perceivers. $(B)$ Left hemispheric brain network differences between high and low perceivers during LO (synchronous and incongruent) condition.

Finally, functional interactions between the PBN and the resting-state networks (RSNs) were estimated by performing a between-network analysis. Pre-processed task-block BOLD time series for the PBN were projected onto their first principal component (Friston et al., 1993) and the same procedure was followed for each RSN: dorsal attention network (DAN), default mode network (DMN), saliency network (SN), fronto-parietal network (FPN), and ventral attention network (VAN), to obtain a lower dimensional representation of network dynamics. Correlation coefficients were computed between temporal projections of the PBN and each RSN for all AV lags and perception-sorted categories. Interestingly, all the RSNs showed high correlation with the PBN, but the correlations got weaker close to AV stimuli synchrony for DAN, DMN, and FPN, and closely resembled the SBC patterns in the case of high perceivers; however, a reversed trend observed for FPN in the case of low perceivers (Fig. 5). Thus, the degree of DAN-PBN and DMN-PBN functional interaction is anticorrelated with propensity to audio-visual illusion for both high and low perceivers. However, a trend of decreasing anti-correlation with increasing illusory perception was observed when the participants are more finely grouped into three categories (SI Appendix, Fig. S12). 
A
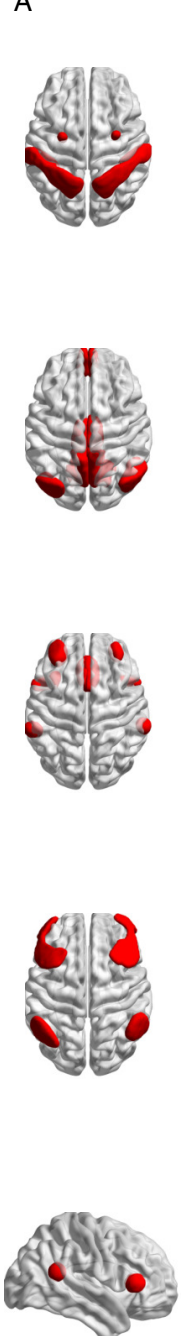
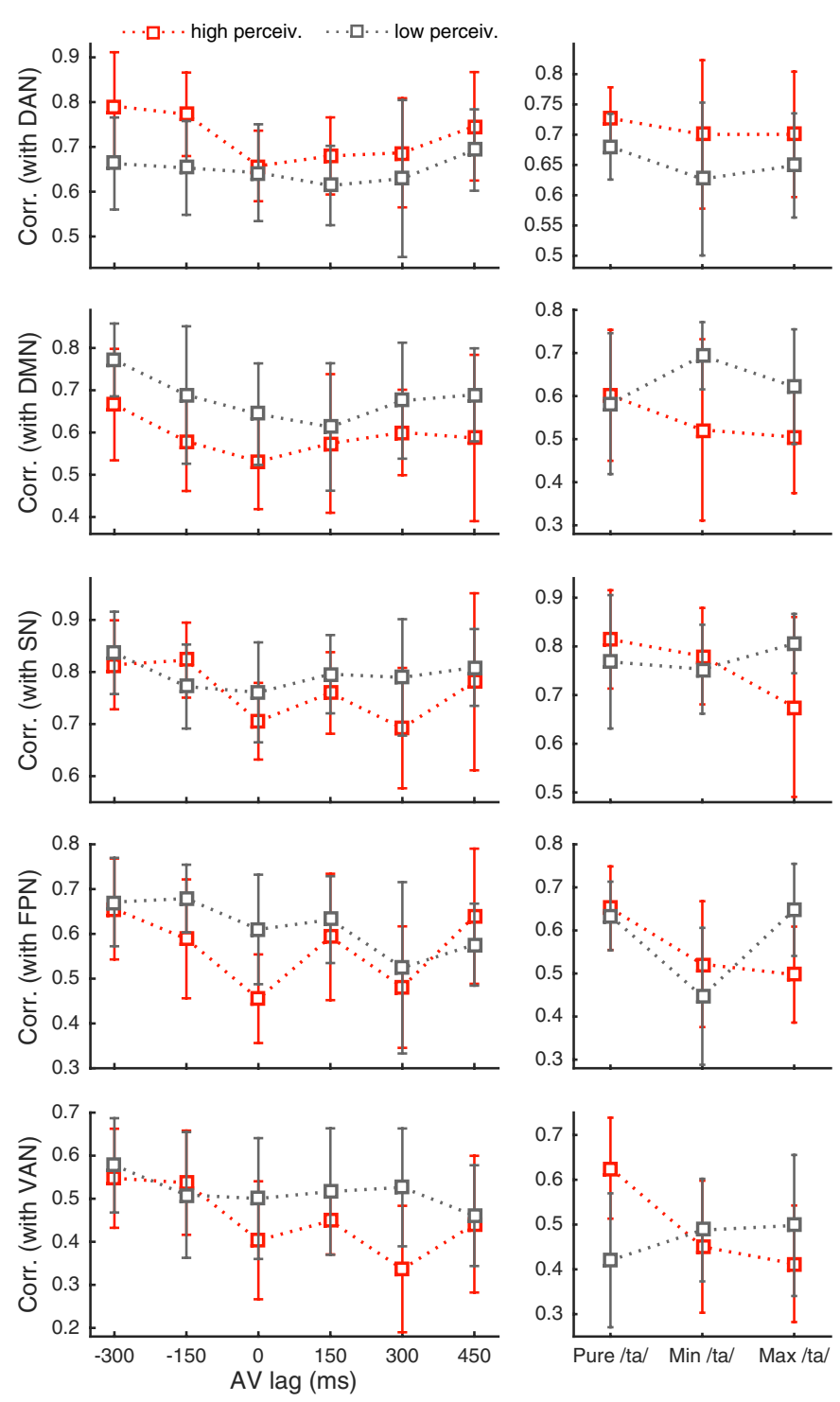

B
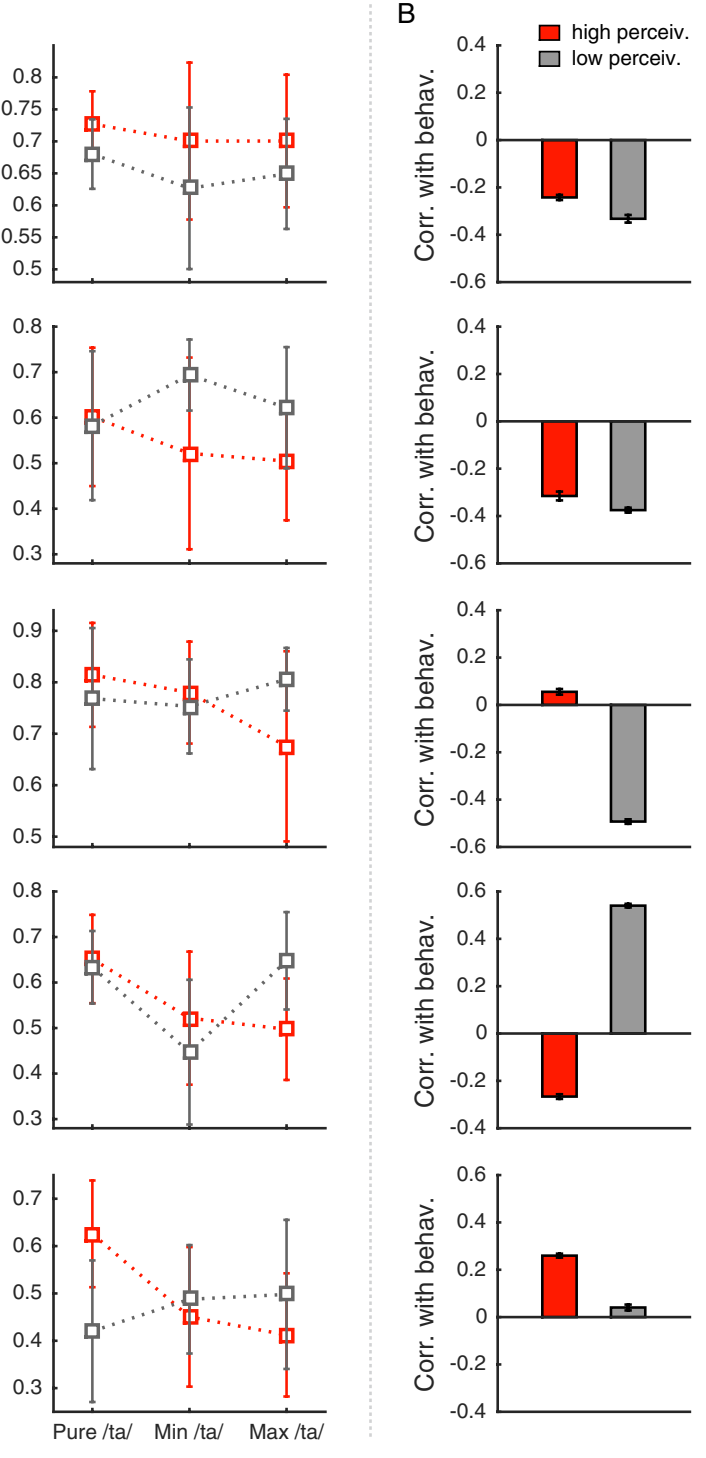

Figure 5. (A) Between-network connectivity computed from projections on first principal component for the perceptual binding network (PBN) and the resting-state brain networks-dorsal attention network (DAN), default mode network (DMN), saliency network (SN), fronto-parietal network (FPN), and ventral attention network (VAN). $(B)$ The correlation between the connectivity trend described in panel $(A)$ and the corresponding trend in propensity to audio-visual illusion across AV lags.

\section{Discussion}

We demonstrated that a homeostatic balance between segregative and integrative information processing among distributed brain areas was critical for the perceptual binding of auditory and visual components of a multisensory stimulus. Enhanced segregated information processing and complementarily reduced integrative processing in each node of the perceptual binding network (PBN) was indicative of higher propensity to perceive illusions. Although the PBN was correlated with restingstate networks, the strengths of the correlation for default mode (DMN) and dorsal attention networks (DAN) decreased during enhancement of cross-modal bindings such as during 0 AV lag or peak of illusory perception. Interestingly, the pattern of decreasing strengths of coupling between nodes of 
PBN and the whole brain (SWFC) was concomitant with rise in illusory perception, i.e., SWFC was anti-correlated with behavior. Follow-up exploration of these patterns in smaller groups divided based on each subject's susceptibility to audio-visual illusion, i.e., in high, medium, and low perceivers, a theory of reduced integrative processing and increased segregative processing for higher cross-modal perception within nodes of PBN gains further ground. Finally, the increased thalamic activity in low perceivers also supported an enhanced role of subcortical level processing in cross-modal perception. (Gau et al., 2020; Noesselt et al., 2010; Tyll et al., 2011). In the following, we discuss these issues in more detail.

Previous studies focused on regions like STS for multisensory processing and integration (Nath and Beauchamp, 2011; Stevenson and James, 2009). However, several unanswered questions related to multisensory integration and more subtly cross-modal binding (how stimulation of one sensory stream affects others) requires a theoretical understanding of distributed network mechanisms (Yau et al., 2015). Our paradigm allowed us to distinguish multisensory processing (involved in all AV lags) from the cross-modal perceptual binding (predominantly in L0 and L1), and the importance of the relationship of pSTS with other distributed regions - together defined as PBN. Following previous studies (Beauchamp, 2005; Beauchamp et al., 2004), our data clearly establishes pSTS activation is enhanced during cross-modal illusory /ta/ perception, compared to similar activation patterns during Pure /ta/ and Min /ta/ perceptual categories. However, as was also observed in L0 stimulus condition where the degree of pSTS activation could not explain the perceptual difference between high and low perceivers (SI Appendix, Figs. S3 and S4, and Tables S1 and S2), the activation as well as interaction among distributed brain areas encompassing the primary sensory areas, motor representaional areas, associative areas such as IPL, V5/MT+, IFG, etc., is essential for audio-visual illusory percept formation. Hence, departing from the earlier studies, our results underscore the need for building an understanding of cross-modal perception at the whole-brain network level to conceptualize the segregated information processing in a large number of cortical areas. Interestingly, we found thalamic activation overall was more dominant in low perceivers which indicated a major role played by the cortico-thalamic circuitry in perceptual binding. Recent studies have demonstrated that decrease in levels of alpha coherence in electroencephalographic (EEG) activity is indicative of propensity to McGurk-illusion perception (Kumar et al., 2020), wherein the cortico-thalamic basis of alpha rhythms is well-known (da Silva et al., 1973) as well as cortico-thalamic influences on early sensory cortices (Noesselt et al., 2010). fMRI time-scale may not allow the temporal resolution to identify the dynamic mechanisms of the cortico-thalamic circuitry, but future electrophysiological studies can surely address this question.

The second important finding of this study was the decrease in correlation between several activated nodal ROIs and rest of the brain areas peaking when maximal cross-modal perceptual binding is reported (Fig. 3 and SI Appendix, Figs. S9 and S10). From the whole-brain network perspectives also, increased cross-modal binding is associated with increased local cohesiveness, balanced by decreased global integration. Subsequently, PBN was also found to be correlated with DAN, DMN, and to some extent $\mathrm{SN}$; but the correlation pattern varied across AV lags reaching a minimum at the peak of illusory perception (Fig. 5). Several researchers have argued for the need to explore the coupling of task-positive networks with task-agnostic networks to understand behavior and inter-subject variability (Fox et al., 2007; Kelly et al., 2008) with some arguing that anti-correlations may reveal the strength of cognitive performance (Hampson et al., 2010). We argue that the competitive balance of integrative and segregative processing delimits an individual's perceptual experience, and indeed alterations of the patterns defined by the individual's functional connectome can explain perceptual variability (Finn et al., 2015). A preliminary attempt in this direction was to divide the participants into three smaller groups - high, medium, and low perceivers - that resulted in higher-to-lower anti- 
correlation between seed functional connectivity and behavior, respectively. High perceivers also displayed relatively higher local clustering and larger characteristic path length (SI Appendix, Fig. S11). To the best of our knowledge, this is the first demonstration of competitive segregation and integration mechanisms in multisensory integration tasks. Some integrative aspects of multisensory processing such as enhanced functional connectivity (FC) among the multisensory and unisensory regions, observed by earlier studies (Nath and Beauchamp, 2011) can be explained from the spatial topographies of functionally connected regions with pSTS (SI Appendix, Figs. S7 and S8) at L0 and L1. Thus, an argument that integrative mechanisms are predominantly compensatory to the taskdriven haemodynamic oxygen metabolism can be put forward while contextualizing our results with previous work on multisensory integration. Temporal binding windows (Stevenson et al., 2012) can define the boundaries of the compensatory regimes where integration and segregation can be synergistically altered, giving rise to inter-trial variability.

The two major findings we presented are also consonant with the theoretical frameworks that are often used to understand multisensory integration, Bayesian causal inference (Koerding et al., 2007; Magnotti et al., 2018; Rohe et al., 2019) and predictive coding hypothesis (Friston and Kiebel, 2009; Mumford, 1991). First, enhanced segregation and decreased integration in distributed brain areas alludes to a mechanism of perceptual binding of disparate sensory objects by imposition of a common causal structure as suggested by earlier studies (Rohe et al., 2019). Second, in harmony with the predictive coding hypothesis, the decreased FC between the context-driven PBN and the contextfree RSNs can be interpreted as signatures of feedback functional projections from hierarchically higher order "dynamic core" (Deco, G. and Kringelbach, M. L. and Jirsa, V. K. and Ritter, P., 2017) that suppresses the SWFC within the temporal binding window. However, our study departs significantly from these earlier theoretical frameworks in providing a more neurobiologically grounded mechanism of multisensory integration based on the homeostatic balance of higher segregation and decreased global integration (Lord et al., 2017) employed by nodes of the PBN. In light of our current results and previous modeling studies (Abeysuriya, R. G. and Hadida, J. and Sotiropoulos, S. N. and Jbabdi, S. and Becker, R. and Hunt, B. A. E. and Brookes, Matthew J. and Woolrich, M. W., 2018; Naskar, Amit and Vattikonda, Anirudh and Deco, Gustavo and Roy, Dipanjan and Banerjee, Arpan, 2021; Vattikonda, A. and Surampudi, B. Raju and Banerjee, A. and Deco, G. and Roy, D., 2016), we propose that homeostatic balance of functional integration and segregation may emerge from the underlying excitatory-inhibitory (E-I) balance in local neuronal populations of involved brain areas and may even be related to neurotransmitter homeostasis (Naskar, Amit and Vattikonda, Anirudh and Deco, Gustavo and Roy, Dipanjan and Banerjee, Arpan, 2021; Shine, J. M. and Müller, E. J and Munn, B. and Cabral, J. and Moran, R. J. and Breakspear, M., 2021). While spontaneous brain activity such as during resting state has been typically associated with the E-I balance (Abeysuriya, R. G. and Hadida, J. and Sotiropoulos, S. N. and Jbabdi, S. and Becker, R. and Hunt, B. A. E. and Brookes, Matthew J. and Woolrich, M. W., 2018; Deco, G. and Kringelbach, M. L. and Jirsa, V. K. and Ritter, P., 2017; Naskar, Amit and Vattikonda, Anirudh and Deco, Gustavo and Roy, Dipanjan and Banerjee, Arpan, 2021; Vattikonda, A. and Surampudi, B. Raju and Banerjee, A. and Deco, G. and Roy, D., 2016), the potential of altered E-I balance-in guiding the formation of perceptual experience from interactions between inclement sensory signals with stored associative memories (Barron, H. C. and Vogels, T. P. and Behrens, T. E. and Ramaswami, M., 2017), as a catalyst for volitional control effectuating perceptual stability (Kondo, H. M. and Pressnitzer, D. and Shimada, Y. and Kochiyama, T. and Kashino, M., 2018), in guiding decision making strategies during multi-attribute choice (Pettine, W. W. and Louie, K. and Murray, J. D. and Wang, X.-J., 2021); all have been proposed recently. According to this line of reasoning, homeostatic balance shift between segregation and integration observed during the illusory perception may be the outcome of an energy consumption by the motor 
networks, reported in an earlier study (Murakami, T. and Abe, M. and Wiratman, W. and Fujiwara, J. and Okamoto, M. and Mizuochi-Endo, T. and Iwabuchi, T. and Makuuchi, M. and Yamashita, A. and Tiksnadi, A. and others, 2018) and supported by underlying neuromodulatory systems which are otherwise adapted to lesser energy consuming congruent auditory-visual input. The existence of the stable illusory perception across groups of people from wide geographical-linguistic backgrounds (Magnotti, J. F. and Mallick, D. B. and Feng, G. and Zhou, B. and Zhou, W. and Beauchamp, M. S., 2015), as well as its robust presence post-learning (Mallick, D. B. and Magnotti, J. F. and Beauchamp, M. S., 2015) also hints toward a neuromodulatory origin. An immediate goal of subsequent studies should be to employ neurostimulation techniques such as TMS and tDCS (Yau et al., 2015) with a goal to perturb the homeostatic balance of integration-segregation mechanisms between PBN and context independent RSNs and identify their deeper causal architecture. Further extending the paradigms and analytic approaches that we have used here, studies on neuropsychiatric and affective disorders can explore whether an alteration of the balance of segregation and integration can be a viable framework to characterize patient-specific symptoms. 


\section{MATERIALS AND METHODS}

\section{Participants and Stimuli.}

Fifty-two healthy right-handed human volunteers ( 25 female, 27 male; age range $=24.5$ years, SD $=3.12$ ) participated in the functional magnetic resonance imaging (fMRI) study. The study was approved by the Institutional Human Ethics Committee (IHEC), National Brain Research Centre, India, and all participants provided informed consents in a format approved by the IHEC.

Seven video stimuli each of 2 second duration were prepared. Six of them were incongruent audio-visual (AV) objects where audio recordings of a male speaker vocalizing $/ \mathrm{pa} /$ is dubbed on a video showing the lip movement while articulating the vocalization of $/ \mathrm{ka} /(/ \mathrm{pa} /-/ \mathrm{ka} /)$. The remaining one video was of a congruent $\mathrm{AV}$ object $(/ \mathrm{ta} /-/ \mathrm{ta} /)$. In the six incongruent videos, the gap between the onset of auditory and visual streams were varied from -300 to $450 \mathrm{~ms}$ in steps of $150 \mathrm{~ms}$ (Fig. 1A). Negative sign indicates that the auditory stimulus onset precedes that of visual lip movement (A leading), and the positive sign on the other hand implies the auditory stimulus succeeds the lip articulation (V leading). An asymmetric range of AV lags were chosen, because Munhall and colleagues (Munhall et al., 1996) reported the dominance of illusory perception toward positive lags where the onset of lip articulation precedes auditory onset. The congruent /ta/-/ta/ stimulus had synchronous onset of AV stimuli. In the stimulus video, the articulation of $/ \mathrm{ka} /$ or $/ \mathrm{ta} /$ always started from a neutral lip-closed position. Videos were created using VideoPad video-editing software (NCH Software, CO, USA) at a frame rate of 25 frames/second and a resolution of $1280 \times 720$ pixels. The auditory /pa/ and /ta/ syllables were of 0.549 and $0.531 \mathrm{~s}$ duration and were edited in Audacity software (Sourceforge.net) to minimize the background noise. The audio sampling rate was $44 \mathrm{kHz}$ and had a bit rate of 128 kbps. The resulted seven stimuli thus have five asynchronous and two synchronous stimuli-McGurk stimuli (/pa/-/ka/) with -300, -150, 0, 150, 300, $450 \mathrm{~ms}$ AV lags, and Pure /ta/ (/ta/-/ta/) stimulus with $0 \mathrm{~ms}$ AV lag. Henceforth, we shall also refer the AV lag conditions in shorter notation as $-\mathrm{L} 2$, $-\mathrm{L} 1, \mathrm{~L} 0, \mathrm{~L} 1, \mathrm{~L} 2$, L3 for $-300,-150,0,150,300,450 \mathrm{~ms}$ lag, respectively.

\section{Task Design.}

The task design is illustrated in Fig. 1A. Inside the scanner, the stimuli were presented in a block design, and each activation block of length 20 s comprised 10 video stimuli of one kind. In total there were 28 activation blocks in the whole experiment: 4 activation blocks for each of the 7 stimulus categories. The 28 activation blocks were alternated with another 28 inter-trial resting blocks, each also of $20 \mathrm{~s}$ duration. We randomized the presentation of the 7 stimulus categories with each category comprising 4 alternated activation blocks of one kind. The stimuli were presented through an INVIVO MR-compatible CRT screen attached to the head-coil and an MRI-compatible headphones (Philips, The Netherlands). Presentation software (Neurobehavioral Systems, CA) was used to display the stimuli. During the presentation, participants were instructed to attend to the AV stimuli watching at the speaker all the times and report their perceptual experience of whether they heard "/pa/", "/ta/," or "any other" through a three-button fibre-optic response device by Curdes (Current Designs, PA, USA), which was used to record the responses of participants. 


\section{fMRI Data Acquisition.}

Structural T1-weighted magnetization-prepared rapid gradient-echo (MP-RAGE) scan was performed in the beginning of each participant's scanning session using a 3 Tesla Philips Achieva MRI scanner. For each participants, seven functional T2* scans $\left(\mathrm{TR}=2 \mathrm{~s}, \mathrm{TE}=35 \mathrm{~ms}\right.$, flip angle $=90^{\circ}$ ) corresponding to the seven different stimuli ( - L2 to L3 and Pure /ta/) were acquired. An 8-channel head coil was used to acquire the T2*-weighted images. The voxel size of the acquired images was $3.59 \times$ $3.59 \times 5 \mathrm{~mm}^{3}$ and the voxel size of the reconstructed images was $1.80 \times 1.80 \times 5 \mathrm{~mm}^{3}$. The slice thickness was $5 \mathrm{~mm}$ and inter-slice distance was $1 \mathrm{~mm}$.

\section{Behavioral Data Analysis.}

The button press responses from the 52 participants were converted into normalized percentage measures of each perceptual response category_/pa/, /ta/, or "other"-separately for each AV lag and Pure /ta/ condition (SI Appendix, Fig. S2A). A maximum of 40 responses were expected from each task. Tasks with less than $80 \%$ responses were rejected, and all participants met this threshold. The volunteers were further screened based on their /ta/ response percentage during congruent Pure /ta/ (/ta/-/ta/) stimulus condition. The minimum threshold of $/ \mathrm{ta} / \mathrm{response}$ was set to $40 \%$, and seven participants were rejected from this criterion. Two more participants were rejected because one had reported $>95 \% / \mathrm{ta} /$ response in five AV lag conditions and another $>95 \%$ "other" response in two AV lag conditions. Thus, only 43 participants were selected based on their behavioral responses. Furthermore, five more participants were rejected because of excessive movement during MRI scanning. Finally, 38 participants were thus selected for further analyses. For each subject the susceptibility to audio-visual illusion was estimated as the average of two maximal percentage $/ \mathrm{ta} / \mathrm{responses}$ at any two AV lags. Based on their susceptibilities, the participants were further sorted into two equalsized groups (19-19): the group representing higher susceptibilities will be called high perceivers and the other at the lower end as low perceivers (SI Appendix, Fig. S1A). Statistical analyses of group responses for each perceptual experience /pa/, /ta/, and "other" were performed using analysis of variance (ANOVA), with lags as independent variable.

\section{fMRI Data Analysis.}

Pre-processing. For each participant, all the acquired functional brain volumes were realigned and co-registered with the structural scan using a six-parameter rigid-body transformation (three translations and three rotations). Those participants with maximum movement greater than 2 units (i.e., 2 $\mathrm{mm}$ in translational axes, or $2^{\circ}$ in rotational axes) in any of the six degrees of freedom were rejected from further analysis. The structural image of each participant's brain was then segmented into grey and white matter maps, and using which the functional brain volumes were spatially normalized to Montréal Neurological Institute (MNI) standard brain. The normalized functional images were then spatially smoothed minimally with a $4 \mathrm{~mm}$ full-width at half-maximum (FWHM) isotropic Gaussian kernel. All the preprocessing was done using Statistical Parametric Mapping Software 8 (SPM8) in MATLAB (MathWorks, MA). The first brain volume of each participant was removed to allow the magnetization to reach to a steady state.

Stimulus-based activation. After pre-processing, we performed the first-level intra-fixed-effects analysis using t-test on the activation blocks versus the inter-trial blocks, with the experimental design (event onset and their time course) as essential regressors and the realignment parameters for 
head movements as nuisance regressors. To accommodate the inter-participant variability within each AV lag and perceptual condition, the contrast images (task block > inter-trial rest block) from the first-level analysis were entered into a second-level random-effects group analysis, using one-sample t-tests. Subsequently, the fMRI data were investigated via two alternative routes. First, the group activation maps (Friston et al., 1995) were extracted for each AV lag, capturing stimulus-driven changes. For each subject we further selected the two AV lags that correspond to maximum and minimum percentage / ta/ responses (Max /ta/ and Min /ta/). Once compiled, the group activation maps in Max $/ \mathrm{ta} /$ and Min $/ \mathrm{ta} /$ were compared against Pure /ta/, thus capturing the brain activations sorted vis-àvis perceptual response. Second, BOLD time series data were extracted for both stimulus-based and perception-based categories, to further estimate the seed-based correlations and other network characteristics (details are provided in the next section). When finally reporting the significantly activated clusters, the identification as well as labeling of the brain areas were done according to the parcels in Brainnetome atlas (Fan et al., 2016) (http://atlas.brainnetome.org/; SI Appendix, Table S3).

For defining seed regions of interest (ROIs), task-active brain parcels were first identified using another subset of participants who reported perceiving illusory /ta/ for at least $60 \%$ of the total responses at any AV lag. Each parcel was located by mapping the task-active clusters during LO and L1 conditions on brain volumes specified with Brainnetome atlas. We particularly chose L0 and L1 AV lag conditions for there were maximal significant activation of brain volumes as well as maximal average illusory /ta/ response during these lags. These active brain parcels were also majorly found to be active during L0, L1, and MaxTa conditions of high and low perceivers. The active parcels were then grouped into 32 ROIs based on division through major anatomical landmarks (SI Appendix, Table S4). The changes in the number of within-ROI task-active voxels for different task conditions were further investigated by creating 19 leave-one-out samples, separately for each high and low perceiver group. Second-level random-effect group analyses were then performed on these 19 subsamples to extract the task-active volume information, and the average of within-ROI activation volume changes were reported in SI Appendix, Figs. S3 and S4.

Percentage signal difference (PSD). For each event in the block design, the ROI percentage signal change was evaluated by estimating maximal height of the HRF fitted event time course (beta $\times$ event regressor), relative to the actual mean signal of the ROI. The signal was estimated for an event duration of 2 seconds. The percentage signal change was extracted using a MarsBaR batch script (http://marsbar.sourceforge.net). For each of the 32 ROIs, the PSD in each AV lag and perceptual condition was then evaluated by subtracting the percentage signal change of inter-trial rest blocks from that of the activation blocks. The changes in average PSD for high and low perceivers across conditions are reported in SI Appendix, Figs. S5 and S6, respectively.

\section{Functional Connectivity Analysis.}

Seed-based functional connectivity. Each participant's grey matter volume was parcellated using the Brainnetome atlas (http://atlas.brainnetome.org/) for the seed-based connectivity analysis. Among the 210 cortical regions of the atlas, the two subregions of pSTS in each hemisphere were merged into a single ROI. In the subcortical region, only the whole thalamic nuclei in each hemisphere were considered. Taken together, the final set of 210 ROIs covered the entire cortical region and the thalamus (SI Appendix, Table S3). For each participant, the mean BOLD time series from each 210 ROI as well as from 32 seed ROIs (SI Appendix, Table S4) were extracted using REX toolbox (Whitfield-Gabrieli, 2009) (https://web.mit.edu/swg/software.htm). From each extracted time series, 
stimulus blocks corresponding to each AV lag and "Pure/ta/" condition were selected based on the experimental design, and then concatenated. The concatenated time series were further preprocessed through linear detrending (linear or linear breakpoint detrending). Functional correlation between each seed region and the remaining of 210 ROIs (i.e., exclusive of seed parcels) were then evaluated for each AV lag and perceptual condition using the Pearson correlation measure (Lee Rodgers and Nicewander, 1988; Pearson, 1895). Finally, the averaged correlation for each seed ROI was evaluated employing Fisher's z-transformed FDR-corrected $(p \leqslant 0.05)$ correlation values. We will term this measure as seed-based whole-brain functional connectivity (SWFC).

The relationship between the trend that SWFC changes across AV lag conditions (Fig. $3 \mathrm{~A}$ and $C$; red and blue lines for the example pSTS ROIs) and that of the changes in the degree of illusory /ta/ perception (Fig. $3 A$ and $C$, gray line) was further evaluated by estimating the Pearson correlation coefficient between the two trends. Hereafter, we will refer it as SWFC-behavior correlation (SBC). Each reported seed SBC is the average significant correlation ( $p \leqslant 0.05$, Bonferroni corrected) value from 50 repeats of 50 bootstrap samples created by random sampling $70 \%$ participants within each category (high and low perceivers). All seed ROIs of high perceivers displayed negative significant $\mathrm{SBC}$, while most of the ROIs in the case of low perceivers showed weak negative or positive SBC (Fig. 3E; SI Appendix, Table S5).

Whole-brain graph theoretic network analysis. For each participant, the functional interaction across all the 210 brain parcels were estimated using pairwise Pearson correlation between corresponding task-block BOLD time series. At the group level, the functional connectomes were generated as follows. First, distinct subsamples were generated by random sampling $70 \%$ of the total participants in each group. For each subsample, the averaged cross-correlation matrix was then evaluated incorporating Fisher's z-transformation, and tested for statistical significance through one-sample ttest. The significant averaged correlations $(p \leqslant 0.05$, FDR corrected) were then applied a threshold of 0.48 to obtain the binarized network matrix; the threshold value of 0.48 was chosen to ensure a statistical power of $\approx 0.9$. Finally, 50 such binarized brain networks were selected for each stimulus (and lag) condition. We also ensured that each selected network form a single connected component to allow comparisons across same network size. Out of the total 210 nodes (210 ROIs), one node in the anterior right parahippocampal area that was frequently disconnected irrespective of stimulus conditions was excluded from the network study. Thus, we generated 50 binarized connected brain networks of size $209 \times 209$ for each group, at each stimulus (and lag) condition. Furthermore, to later estimate normalized network parameters, for each generated binarized brain network we further generated 50 randomized connected network samples with same degree sequence. We used the MATLAB script randmio_und_connected.m available with Brain Connectivity Toolbox (BCT) for the randomization, by keeping edge iterations to 30 (Rubinov, M. and Sporns, O., 2010).

To study the functional integration and segregation at different scales of brain network, we calculated average local clustering coefficient $\left(C_{\text {real }}\right)$, characteristics path length $\left(L_{\text {real }}\right)$, modularity $\left(Q_{\text {real }}\right)$, and node participation coefficients $(P) . C_{\text {real }}$ and $L_{\text {real }}$ estimate the network characteristics at the resolution of nodes (local scale), and $Q_{\text {real }}$ and $P$ estimates at the level of the entire network (global scale). In this study, we used Blondel et al. (Blondel, V. D and Guillaume, J.-L. and Lambiotte, R. and Lefebvre, E., 2008) method (Louvain method; community_louvain.m available with BCT) for patitioning a network into nonoverlapping functional modules. We also applied normalized graph measures for comparing across the networks (Guimera, R. and Sales-Pardo, M. and Amaral, L. A. N., 2007; Van Wijk et al., 2010).

The local clustering coefficient of a node estimates the fraction of connections among the first neighbors of the node against all possible connection, and the normalized averaged clustering coeffi- 
cient $\left(C_{n}\right)$ is defined as

$$
C_{n}=\frac{C_{\text {real }}}{C_{\text {rand }}}
$$

where $C_{\text {real }}$ and $C_{\text {rand }}$ are the average local clustering coefficients (Rubinov, M. and Knock, S. A. and Stam, C. J. and Micheloyannis, S. and Harris, A. W. F. and Williams, L. M. and Breakspear, M., 2009; Rubinov, M. and Sporns, O., 2010) of the empirical and randomized networks, respectively.

Similarly, for the characteristics path length that measures the average shortest path across all node pairs, the normalized characteristics path length is

$$
L_{n}=\frac{L_{\text {real }}}{L_{\text {rand }}}
$$

where $L_{\text {real }}$ and $L_{\text {rand }}$ represent the characteristics path length (Rubinov, M. and Sporns, O., 2010) of the empirical and randomized networks, respectively.

Modularity on the other hand is a quality function that measures how well a network is divided into distinct functional units, and it can be conceptualized as another network segregation measure at a larger functional modular scale (Newman, M. E. J. and Girvan, M., 2004). The normalized modularity is further estimated as (Kashtan, N. and Alon, U., 2005)

$$
Q_{n}=\frac{Q_{\text {real }}-Q_{\text {rand }}}{Q_{\max }-Q_{\text {rand }}}
$$

Here $Q_{\max }$ is the maximal possible network modularity value with same degree sequence and can be approximated as $Q_{\max }=1-1 / m$, where $m$ represents the total number of modules in the real network (Parter, M. and Kashtan, N. and Alon, U., 2007). $Q_{\text {real }}$ and $Q_{\text {rand }}$ are modularity computed in the empirical and randomized network (Newman, M. E. J. and Girvan, M., 2004) respectively.

Node participation coefficient $(P)$ measures how equally the connections of a node are distributed among all available modules of a network (Guimera, Roger and Amaral, Luis A Nunes, 2005). Thus, it estimates the capacity of a node in intermodular information integration.

$$
P=1-\sum_{i=1}^{m}\left(\frac{k_{i}}{k}\right)^{2}
$$

where $k$ is the degree of the node and $k_{i}$ represents the number of connections the node have with the module $i$. Here, $m$ is the number of available modules.

Interaction between the PBN and resting-state networks (RSNs). We also investigated the functional interaction between the PBN and human default mode network (DMN), saliency network (SN), fronto-parietal network (FPN), dorsal attention network (DAN), and ventral attention network (VAN). The ROI masses for DMN, SN, FPN, and DAN were taken from CONN toolbox (WhitfieldGabrieli and Nieto-Castanon, 2012) which elucidated these RSNs through ICA analyses of 497 participants from HCP dataset (www.nitrc.org/projects/conn). For the VAN, two spherical ROI masses each of $10 \mathrm{~mm}$ diameter were created using MarsBaR toolbox: one for the right temporo-parietal junction (Fox et al., 2006) (centered around MNI coordinates: 54, -50, 19) and another for the right ventrolateral prefrontal cortex (Sylvester et al., 2013) (centered around MNI coordinates: 51, 27, 8). Task-block BOLD time series for the respective ROIs were extracted using REX toolbox. Next, through principal component decomposition the projected time series from each network constituents to their first principal component was evaluated. Finally, network interactions were estimated by using Pearson correlation measure between corresponding first principal component time series. We reported the evolution of the network interaction across AV lag and perceptual conditions in Fig. 5. 


\section{References}

Abeysuriya, R. G. and Hadida, J. and Sotiropoulos, S. N. and Jbabdi, S. and Becker, R. and Hunt, B. A. E. and Brookes, Matthew J. and Woolrich, M. W. A biophysical model of dynamic balancing of excitation and inhibition in fast oscillatory large-scale networks. PLoS Comput. Biol., 14:e1006007, Feb. 2018. ISSN 1553-7358. doi: 10.1371/journal.pcbi.1006007.

D. Alais and D. Burr. The ventriloquist effect results from near-optimal bimodal integration. Curr. Biol., 14:257-262, 2004.

Barron, H. C. and Vogels, T. P. and Behrens, T. E. and Ramaswami, M. Inhibitory engrams in perception and memory. Proc. Natl. Acad. Sci. U.S.A., 114:6666-6674, June 2017. ISSN 10916490. doi: 10.1073/pnas.1701812114.

M. S. Beauchamp. See me, hear me, touch me: multisensory integration in lateral occipital-temporal cortex. Curr. Opin. Neurobiol., 15:145-153, 2005.

M. S. Beauchamp, B. D. Argall, J. Bodurka, J. H. Duyn, and A. Martin. Unraveling multisensory integration: patchy organization within human STS multisensory cortex. Nat. Neurosci., 7:11901192, Nov. 2004. ISSN 1097-6256. doi: 10.1038/nn1333.

P. Bertelson and G. Aschersleben. Temporal ventriloquism: crossmodal interaction on the time dimension. 1. Evidence from auditory-visual temporal order judgment. Int. J. Psychophysiol., 50: 147-155, Oct. 2003. ISSN 0167-8760. doi: 10.1016/s0167-8760(03)00130-2.

Blondel, V. D and Guillaume, J.-L. and Lambiotte, R. and Lefebvre, E. Fast unfolding of communities in large networks. J. Stat. Mech., 2008(10):P10008, 2008.

J. R. Cohen and M. D'Esposito. The segregation and integration of distinct brain networks and their relationship to cognition. J. Neurosci., 36:12083-12094, Nov. 2016. ISSN 1529-2401. doi: 10.1523/JNEUROSCI.2965-15.2016.

C. Cuppini, L. Shams, E. Magosso, and M. Ursino. A biologically inspired neurocomputational model for audiovisual integration and causal inference. Eur. J. Neurosci., 46:2481-2498, Nov. 2017. ISSN 1460-9568. doi: 10.1111/ejn.13725.

F. H. L. da Silva, T. H. M. T. van Lierop, C. F. Schrijer, and W. S. van Leeuwen. Organization of thalamic and cortical alpha rhythms: Spectra and coherences. Elec. Clin. Neuro., 3(6):627 - 639, 1973.

Deco, G. and Kringelbach, M. L. and Jirsa, V. K. and Ritter, P. The dynamics of resting fluctuations in the brain: metastability and its dynamical cortical core. Sci. Rep., 7:3095, June 2017. ISSN 2045-2322. doi: 10.1038/s41598-017-03073-5.

L. Fan, H. Li, J. Zhuo, Y. Zhang, J. Wang, L. Chen, Z. Yang, C. Chu, S. Xie, A. R. Laird, P. T. Fox, S. B. Eickhoff, C. Yu, and T. Jiang. The human Brainnetome Atlas: a new brain atlas based on connectional architecture. Cereb. Cortex, 26:3508-3526, Aug. 2016. ISSN 1460-2199. doi: 10.1093/cercor/bhw157. 
E. S. Finn, X. Shen, D. Scheinost, M. D. Rosenberg, J. Huang, M. M. Chun, X. Papademetris, and R. T. Constable. Functional connectome fingerprinting: identifying individuals using patterns of brain connectivity. Nat. Neurosci., 18:1664-1671, Nov. 2015. ISSN 1546-1726. doi: 10.1038/nn.4135.

M. D. Fox, M. Corbetta, A. Z. Snyder, J. L. Vincent, and M. E. Raichle. Spontaneous neuronal activity distinguishes human dorsal and ventral attention systems. Proc. Natl. Acad. Sci. U.S.A., 103(26): 10046-10051, 2006.

M. D. Fox, A. Z. Snyder, J. L. Vincent, and M. E. Raichle. Intrinsic fluctuations within cortical systems account for intertrial variability in human behavior. Neuron, 56:171-184, Oct. 2007. ISSN 0896-6273. doi: 10.1016/j.neuron.2007.08.023.

K. Friston and S. Kiebel. Predictive coding under the free-energy principle. Philos. Trans. R. Soc. Lond. B Biol. Sci., 364:1211-1221, May 2009. ISSN 1471-2970. doi: 10.1098/rstb.2008.0300.

K. J. Friston, C. D. Frith, P. F. Liddle, and R. S. Frackowiak. Functional connectivity: the principalcomponent analysis of large (PET) data sets. J. Cereb. Blood Flow Metab., 13:5-14, Jan. 1993. ISSN 0271-678X. doi: 10.1038/jcbfm.1993.4.

K. J. Friston, A. P. Holmes, K. J. Worsley, J.-P. Poline, C. D. Frith, and R. S. J. Frackowiak. Statistical parametric maps in functional imaging: a general linear approach. Hum. Brain Mapp., 2:189-210, 1995. ISSN 1065-9471. doi: 10.1002/hbm.460020402.

R. Gau, P.-L. Bazin, R. Trampel, R. Turner, and U. Noppeney. Resolving multisensory and attentional influences across cortical depth in sensory cortices. Elife, 9:e46856, 2020.

Guimera, Roger and Amaral, Luis A Nunes. Functional cartography of complex metabolic networks. Nature, 433(7028):895-900, 2005.

M. Hampson, N. Driesen, J. K. Roth, J. C. Gore, and R. T. Constable. Functional connectivity between task-positive and task-negative brain areas and its relation to working memory performance. Magn. Reson. Imaging, 28:1051-1057, Oct. 2010. ISSN 1873-5894. doi: 10.1016/j.mri.2010.03.021.

J. A. Jones and D. E. Callan. Brain activity during audiovisual speech perception: an fMRI study of the McGurk effect. Neuroreport, 14:1129-1133, June 2003. ISSN 0959-4965. doi: 10.1097/00001756-200306110-00006.

Kashtan, N. and Alon, U. Spontaneous evolution of modularity and network motifs. Proc. Natl. Acad. Sci. U.S.A., 102(39):13773-13778, 2005.

A. M. C. Kelly, L. Q. Uddin, B. B. Biswal, F. X. Castellanos, and M. P. Milham. Competition between functional brain networks mediates behavioral variability. Neuroimage, 39:527-537, Jan. 2008. ISSN 1053-8119. doi: 10.1016/j.neuroimage.2007.08.008.

K. P. Koerding, U. Beierholm, W. J. Ma, S. Quartz, J. B. Tenenbaum, and L. Shams. Causal inference in multisensory perception. PLoS One, 2:e943, Sept. 2007. ISSN 1932-6203. doi: 10.1371/journal.pone.0000943.

Kondo, H. M. and Pressnitzer, D. and Shimada, Y. and Kochiyama, T. and Kashino, M. Inhibitionexcitation balance in the parietal cortex modulates volitional control for auditory and visual multistability. Sci. Rep., 8:14548, Sept. 2018. ISSN 2045-2322. doi: 10.1038/s41598-018-32892-3. 
G. V. Kumar, S. Dutta, S. Talwar, D. Roy, and A. Banerjee. Biophysical mechanisms governing large-scale brain network dynamics underlying individual-specific variability of perception. Eur. $J$. Neurosci., Apr. 2020. ISSN 1460-9568. doi: 10.1111/ejn.14747.

J. Lee Rodgers and W. A. Nicewander. Thirteen ways to look at the correlation coefficient. Am. Stat., 42(1):59-66, 1988.

L.-D. Lord, A. B. Stevner, G. Deco, and M. L. Kringelbach. Understanding principles of integration and segregation using whole-brain computational connectomics: implications for neuropsychiatric disorders. Philos. Trans. A Math. Phys. Eng. Sci., 375:20160283, June 2017. ISSN 1364-503X. doi: 10.1098/rsta.2016.0283.

J. F. Magnotti, K. B. Smith, M. Salinas, J. Mays, L. L. Zhu, and M. S. Beauchamp. A causal inference explanation for enhancement of multisensory integration by co-articulation. Sci. Rep., 8:18032, Dec. 2018. ISSN 2045-2322. doi: 10.1038/s41598-018-36772-8.

Magnotti, J. F. and Mallick, D. B. and Feng, G. and Zhou, B. and Zhou, W. and Beauchamp, M. S. Similar frequency of the McGurk effect in large samples of native Mandarin Chinese and American English speakers. Exp. Brain Res., 233:2581-2586, Sept. 2015. ISSN 1432-1106. doi: 10.1007/s00221-015-4324-7.

Mallick, D. B. and Magnotti, J. F. and Beauchamp, M. S. Variability and stability in the McGurk effect: contributions of participants, stimuli, time, and response type. Psychon. Bull. Rev., 22: 1299-1307, Oct. 2015. ISSN 1531-5320. doi: 10.3758/s13423-015-0817-4.

H. McGurk and J. Macdonald. Hearing lips and seeing voices. Nature, 264:746-748, 1976.

M. A. Meredith and B. E. Stein. Interactions among converging sensory inputs in the superior colliculus. Science, 221(4608):389-391, Jul 1983.

H. Mohr, U. Wolfensteller, R. F. Betzel, B. Misic, O. Sporns, J. Richiardi, and H. Ruge. Integration and segregation of large-scale brain networks during short-term task automatization. Nat. Commun., 7:13217, Nov. 2016. ISSN 2041-1723. doi: 10.1038/ncomms13217.

S. Molholm, W. Ritter, M. M. Murray, D. C. Javitt, C. E. Schroeder, and J. J. Foxe. Multisensory auditory-visual interactions during early sensory processing in humans: a high-density electrical mapping study. Brain Res. Cogn. Brain Res., 14:115-128, June 2002. ISSN 0926-6410. doi: 10.1016/s0926-6410(02)00066-6.

D. Mumford. On the computational architecture of the neocortex. I. The role of the thalamo-cortical loop. Biol. Cybern., 65:135-145, 1991. ISSN 0340-1200. doi: 10.1007/BF00202389.

K. G. Munhall, P. Gribble, L. Sacco, and M. Ward. Temporal constraints on the McGurk effect. Atten. Percept. Psychophys., 58:351-362, 1996.

Naskar, Amit and Vattikonda, Anirudh and Deco, Gustavo and Roy, Dipanjan and Banerjee, Arpan. Multi-scale dynamic mean field model (MDMF) relates resting-state brain dynamics with local cortical excitatory-inhibitory neurotransmitter homeostasis. Netw. Neurosci., pages 1-55, 2021.

A. R. Nath and M. S. Beauchamp. Dynamic changes in superior temporal sulcus connectivity during perception of noisy audiovisual speech. J. Neurosci., 31(5):1704-1714, 2011. 
Newman, M. E. J. and Girvan, M. Finding and evaluating community structure in networks. Phys. Rev. E, 69(2):026113, 2004.

T. Noesselt, S. Tyll, C. N. Boehler, E. Budinger, H.-J. Heinze, and J. Driver. Sound-induced enhancement of low-intensity vision: multisensory influences on human sensory-specific cortices and thalamic bodies relate to perceptual enhancement of visual detection sensitivity. J. Neurosci., 30(41):13609-13623, 2010.

Parter, M. and Kashtan, N. and Alon, U. Environmental variability and modularity of bacterial metabolic networks. BMC Evol. Biol., 7(1):1-8, 2007.

K. Pearson. Vii. note on regression and inheritance in the case of two parents. Proc. R. Soc. Lond., 58(347-352):240-242, 1895.

Pettine, W. W. and Louie, K. and Murray, J. D. and Wang, X.-J. Excitatory-inhibitory tone shapes decision strategies in a hierarchical neural network model of multi-attribute choice. PLoS Comput. Biol., 17:e1008791, Mar. 2021. ISSN 1553-7358. doi: 10.1371/journal.pcbi.1008791.

T. Rohe, A.-C. Ehlis, and U. Noppeney. The neural dynamics of hierarchical Bayesian causal inference in multisensory perception. Nat. Commun., 10:1907, Apr. 2019. ISSN 2041-1723. doi: 10.1038/s41467-019-09664-2.

Rubinov, M. and Knock, S. A. and Stam, C. J. and Micheloyannis, S. and Harris, A. W. F. and Williams, L. M. and Breakspear, M. Small-world properties of nonlinear brain activity in schizophrenia. Hum. Brain Mapp., 30(2):403-416, 2009.

Rubinov, M. and Sporns, O. Complex network measures of brain connectivity: uses and interpretations. Neuroimage, 52(3):1059-1069, 2010.

L. Shams, Y. Kamitani, and S. Shimojo. Visual illusion induced by sound. Brain. Res. Cogn. Brain. Res., 14:147-152, 2002.

Shine, J. M. and Müller, E. J and Munn, B. and Cabral, J. and Moran, R. J. and Breakspear, M. Computational models link cellular mechanisms of neuromodulation to large-scale neural dynamics. Nat. Neurosci., pages 1-12, 2021.

R. A. Stevenson and T. W. James. Audiovisual integration in human superior temporal sulcus: inverse effectiveness and the neural processing of speech and object recognition. Neuroimage, 44:12101223, Feb. 2009. ISSN 1095-9572. doi: 10.1016/j.neuroimage.2008.09.034.

R. A. Stevenson, R. K. Zemtsov, and M. T. Wallace. Individual differences in the multisensory temporal binding window predict susceptibility to audiovisual illusions. J. Exp. Psychol. Hum. Percept. Perform., 38:1517-1529, Dec. 2012. ISSN 1939-1277. doi: 10.1037/a0027339.

C. M. Sylvester, D. M. Barch, M. Corbetta, J. D. Power, B. L. Schlaggar, and J. L. Luby. Resting state functional connectivity of the ventral attention network in children with a history of depression or anxiety. J. Am. Acad. Child Adolesc. Psychiatry, 52(12):1326-1336, 2013.

Dehaene-Lambertz, Ghislaine and Hertz-Pannier, Lucie and Dubois, Jessica and Mériaux, Sébastien and Roche, Alexis and Sigman, Mariano and Dehaene, Stanislas. Functional organization of perisylvian activation during presentation of sentences in preverbal infants. Proc. Natl. Acad. Sci. U.S.A., 103(38):14240-14245, 2006. 
Guimera, R. and Sales-Pardo, M. and Amaral, L. A. N. Classes of complex networks defined by role-to-role connectivity profiles. Nat. Phys., 3(1):63-69, 2007.

Liberman, Alvin M and Whalen, Doug H. On the relation of speech to language. Trends Cogn. Sci., 4(5):187-196, 2000.

Morís Fernández, L. and Macaluso, E. and Soto-Faraco, S. Audiovisual integration as conflict resolution: the conflict of the McGurk illusion. Hum. Brain Mapp., 38(11):5691-5705, 2017.

Murakami, T. and Abe, M. and Wiratman, W. and Fujiwara, J. and Okamoto, M. and Mizuochi-Endo, T. and Iwabuchi, T. and Makuuchi, M. and Yamashita, A. and Tiksnadi, A. and others. The motor network reduces multisensory illusory perception. J. Neurosci., 38(45):9679-9688, 2018.

Scott, S. K and McGettigan, C. and Eisner, F. A little more conversation, a little less action-candidate roles for the motor cortex in speech perception. Nat. Rev. Neurosci., 10(4):295-302, 2009.

B. Thakur, A. Mukherjee, A. Sen, and A. Banerjee. A dynamical framework to relate perceptual variability with multisensory information processing. Sci. Rep., 6:31280, Aug. 2016. ISSN 20452322. doi: $10.1038 / \mathrm{srep} 31280$.

D. Tomasi, R. Wang, G.-J. Wang, and N. D. Volkow. Functional connectivity and brain activation: a synergistic approach. Cereb. Cortex, 24:2619-2629, Oct. 2014. ISSN 1460-2199. doi: 10.1093/cercor/bht119.

G. Tononi, O. Sporns, and G. M. Edelman. A measure for brain complexity: relating functional segregation and integration in the nervous system. Proc. Natl. Acad. Sci. U.S.A., 91:5033-5037, May 1994. ISSN 0027-8424. doi: 10.1073/pnas.91.11.5033.

S. Tyll, E. Budinger, and T. Noesselt. Thalamic influences on multisensory integration. Commun. Integr. Biol., 4(4):378-381, 2011.

B. C. Van Wijk, C. J. Stam, and A. Daffertshofer. Comparing brain networks of different size and connectivity density using graph theory. PloS One, 5(10):e13701, 2010.

Vattikonda, A. and Surampudi, B. Raju and Banerjee, A. and Deco, G. and Roy, D. Does the regulation of local excitation-inhibition balance aid in recovery of functional connectivity? A computational account. Neuroimage, 136:57-67, Aug. 2016. ISSN 1095-9572. doi: 10.1016/j.neuroimage.2016.05.002.

S. Watkins, L. Shams, O. Josephs, and G. Rees. Activity in human V1 follows multisensory perception. Neuroimage, 37:572-578, Aug. 2007. ISSN 1053-8119. doi: 10.1016/j.neuroimage.2007.05.027.

S. Whitfield-Gabrieli. Region of interest extraction (rex) toolbox. Boston, MA, 497, 2009.

S. Whitfield-Gabrieli and A. Nieto-Castanon. Conn: a functional connectivity toolbox for correlated and anticorrelated brain networks. Brain Connect., 2(3):125-141, 2012.

J. M. Yau, G. C. DeAngelis, and D. E. Angelaki. Dissecting neural circuits for multisensory integration and crossmodal processing. Philos. Trans. R. Soc. Lond. B Biol. Sci., 370:20140203, Sept. 2015. ISSN 1471-2970. doi: 10.1098/rstb.2014.0203. 
Zopf Jr, G. W. Sensory Homeostasis. In Progress in Brain Research, volume 2, pages 114-121. Elsevier, 1963.

Acknowledgments This study was supported by NBRC Core funds and Computing Facility. AB was supported by Ramalingaswami Fellowship (BT/RLF/Re-entry/31/2011) and Innovative Young Biotechnologist Award (IYBA, BT/07/IYBA/2013), both from Department of Biotechnology, Ministry of Science and Technology, Government of India. SSS was supported by a National Post-Doctoral Fellowship from Science and Engineering Research Board, Government of India (SERB-NPDF; No. PDF/2016/003188).

Competing interests The authors declare no competing interest. 